\title{
UNA NUEVA ESPECIE DE MAMMILLARIA (CACTACEAE) PARA TAMAULIPAS, MÉXICO
}

\author{
José Guadalupe Martínez-Ávalos ${ }^{1,5}$, Jordan Golubov ${ }^{2}$, Salvador Arias ${ }^{3}$ Y José \\ Ángel Villarreal-Quintanilla ${ }^{4}$
}

${ }^{1}$ Universidad Autónoma de Tamaulipas, Instituto de Ecología Aplicada, División del Golfo 356, Colonia Libertad, 87019 Cd. Victoria, Tamaulipas, México. ${ }^{2}$ Universidad Autónoma Metropolitana, Unidad Xochimilco, Departamento El Hombre y su Ambiente, Calzada del Hueso 1100, Colonia Villa Quietud, 04960 Coyoacán, México, D.F., México.

${ }^{3}$ Universidad Nacional Autónoma de México, Instituto de Biología, Jardín Botánico, Circuito Exterior, Ciudad Universitaria, 04510 México D.F., México. ${ }^{4}$ Universidad Autónoma Agraria Antonio Narro, Departamento de Botánica, Antonio Narro 1923, Buenavista 25315 Saltillo, Coahuila, México. 5Autor para la correspondencia: jmartin@uat.edu.mx

\section{RESUMEN}

Se propone a Mammillaria cielensis (Cactaceae), como una especie nueva de la Reserva de la Biosfera "El Cielo" (RBC) en Tamaulipas, México. Es morfológicamente muy similar a M. zublerae Repp., de la cual difiere en el tamaño del tallo, la forma redondeada del ápice, los tubérculos de menor tamaño y glabros, aréolas con lana blanca, las espinas radiales y centrales más numerosas y ligeramente más cortas, frutos de diferente color y tamaño, la semilla de mayor tamaño, con testa porosa y el hilo de mayores dimensiones. $\mathrm{Su}$ distribución conocida se restringe al bosque de pino encino en el área de la RBC, al sur del estado de Tamaulipas, México. Se presenta una comparación con las especies afines.

Palabras clave: Cactaceae, Mammillaria, México, Tamaulipas, taxonomía.

\section{ABSTRACT}

Mammillaria cielensis (Cactaceae), a new species of Cactaceae from "El Cielo" Biosphere Reserve (RBC) in Tamaulipas, Mexico, is described and illustrated. The new 
species is similar to $M$. zublerae Repp., differing from this species in the size of the stem, the rounded form of the apex, the smaller tubercles, the areoles with white wool, the more numerous and somewhat shorter radial and central spines, the colour and size of the fruits, the shinier and larger seeds, with heavily pitted and slightly reticulate testa, and larger hilum. Its known distribution is restricted to pine oak forest in the $\mathrm{RBC}$, in the southern part of the state of Tamaulipas, Mexico. A comparison with related species is presented.

Key words: Cactaceae, Mammillaria, Mexico, Tamaulipas, taxonomy.

Los géneros Opuntia y Mammillaria son de los más diversos a nivel de especie en la familia Cactaceae (Bravo y Sánchez-Mejorada, 1991; Butterworth y Wallace, 2004; Guzmán et al., 2003; Lüthy, 1995; 2001; Pilbeam, 1999), especialmente en México que es el centro de diversificación de ambos. Se realizaron numerosas colectas de este grupo de plantas durante 1995 para estimar la diversidad de la familia en los diferentes ambientes de la Reserva de la Biosfera "El Cielo" en el estado de Tamaulipas, México (Martínez-Ávalos et al., 1996). Durante los recorridos se encontraron varios sitios donde existe, al parecer, una especie aún desconocida de Mammillaria asociada al subgénero Mammillaria, serie Proliferae D.R. Hunt. En particular se trata de un taxon fuertemente relacionado con M. zublerae Repp.

M. cielensis se compara con M. zublerae, tomando en cuenta la descripción original de este taxon hecha por Reppenhagen (1991-1992), así como la incluida en las revisiones del género realizadas por Hunt et al. (2006) y Lüthy $(1995,2001)$. Se coteja además con M. picta Meinsh, M. vetula Mart. y M. prolifera (Mill.) Haw, dada la afinidad morfológica y filogenética que muestra con este grupo de plantas (Hunt, 1997; Reppenhagen, 1991-1992; Butterworth y Wallace, 2004).

Mammillaria cielensis Martínez-Ávalos, J. Golubov, S. Arias et Villarreal, sp. nov. (Fig. 1).

Planta simplex vel caespitosa, spinis albis, tuberculis sine trichomatibus, floribus flavis, seminibus ellipticis cum testa valde foveolata, leviter reticulata, nigris, nitentissimis, hilo magno, ventrali vel sub-basali.

Planta simple a muy cespitosa y formando grupos numerosos, rupícola; tallo globoso a oviforme, de 24-38 mm de altura (longitud) y 35-37 mm de ancho (diámetro), cubierto por espinas, ápice redondeado; tubérculos cilíndricos, de 6-8 $\mathrm{mm}$ de 
Martínez-Ávalos et al.: Nueva especie de Mammillaria para Tamaulipas, México
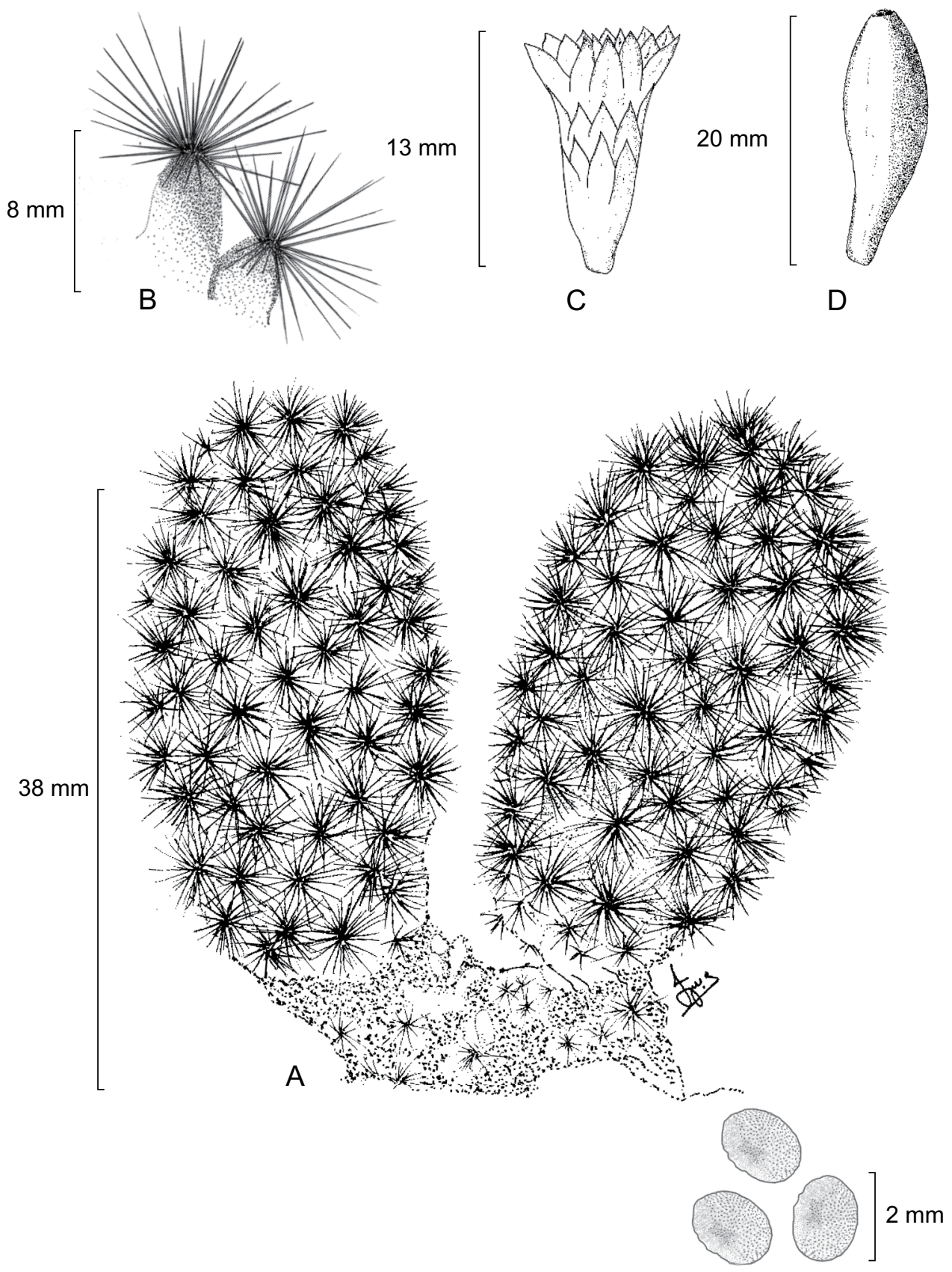

Fig. 1. Esquema de Mammillaria cielensi. mostrando: a. la planta; b. los tubérculos; c. la flor; d. el fruto y e. las semillas (Ilustración de José Guadalupe Martínez Ávalos). 
largo (longitud) y de 4-4.5 mm de ancho, erectos, con jugo acuoso, verdes oscuros, sin tricomas en las axilas; aréolas circulares con lana blanca, de 1.5-2 mm de diámetro, con tricomas muy cortos y blancos; espinas radiales 25-27, de 6-7 mm de longitud, aciculares, de color blanco vítreo, flexibles, siempre cubriendo los tubérculos, espinas centrales 10-12, hasta de $7 \mathrm{~mm}$ de longitud, aciculares, de color similar a las radiales. Flor infundibuliforme, de $12-13 \mathrm{~mm}$ de largo y $10-14 \mathrm{~mm}$ de ancho; el perianto sin abrirse por completo, pericarpelos de $3 \mathrm{~mm}$ de largo y 12 de ancho, de color amarillo pálido; segmentos externos de 8 de $11 \mathrm{~mm}$ de largo, $2.5 \mathrm{~mm}$ de ancho, ápice casi redondeado, amarillos; segmentos internos 23, de $6 \mathrm{~mm}$ de largo y 2.5 $\mathrm{mm}$ de ancho, amarillos; estambres numerosos, de $5 \mathrm{~mm}$ de largo, de color amarillo pálido; estilo de $11 \mathrm{~mm}$ de largo, amarillento, los lóbulos del estigma 4-5, de $2.5 \mathrm{~mm}$ de largo, de color amarillento. Fruto claviforme, de 10-20 mm de largo y ca. 3.5-4 $\mathrm{mm}$ de ancho, rojo a rosado, con pocas semillas; éstas de forma elíptica, de ca. $2 \mathrm{~mm}$ de largo y $1.7 \mathrm{~mm}$ de ancho, testa foveolada muy hundida, ligeramente reticulada, negra brillante, hílum de $52 \mu \mathrm{m}$ de longitud, ventral a subbasal.

Tipo: México. Tamaulipas: municipio de Jaumave, al norte de la Reserva de la Biosfera "El Cielo", 1900 m s.n.m., 20 de febrero de 2001, José G. Martínez-Ávalos 890 (Holotipo: UAT; isotipos por enviar: ANSM, MEXU, UT).

Material adicional examinado: Tamaulipas: entre los municipios de Jaumave y Llera, zona norte de la Reserva de la Biosfera "El Cielo", 1840 m, 7 de abril de 2009, José G. Martínez-Ávalos 1560 (UAT); 27 km al noroeste de Ocampo, sobre la carretera a Tula, José G. Martínez-Ávalos 1780 (UAT); $5 \mathrm{~km}$ al este de Joya de Salas, municipio de Jaumave, José G. Martínez-Ávalos 1781 (UAT); 4 km al norte del Julilo, municipio de Llera, José G. Martínez-Ávalos, 1782 (UAT).

Distribución y hábitat. Mammillaria cielensis habita en la parte norte de la Reserva de la Biosfera "El Cielo", en el estado de Tamaulipas. Por lo general crece formando colonias aisladas sobre rocas metamórficas con suelos oscuros ricos en materia orgánica. La vegetación corresponde a bosques de pino-encino (Puig y Bracho, 1987) y el clima es del tipo $\mathrm{C}\left(\mathrm{w}_{0}\right)$ templado subhúmedo con lluvias en verano (Anónimo, 2001). La precipitación del mes más seco es menor de $40 \mathrm{~mm}$, mientras que la invernal fluctúa entre 5 y $10.2 \%$. La temperatura promedio para la zona se encuentra entre 18 y $20^{\circ} \mathrm{C}$ y la precipitación promedio anual oscila entre los 700 y $800 \mathrm{~mm}$ (datos de la estación meteorológica Gómez Farías) (Anónimo, 2002). La mayoría de las poblaciones observadas crece en sitios rocosos sombreados con ex- 
posición norte, ocasionalmente en lugares abiertos con poca vegetación, en altitudes que van de 1700 a $1900 \mathrm{~m}$ (Fig. 2).

Fenología. Florece durante los meses de enero y febrero, y es a principios de marzo cuando sus frutos regularmente empiezan a madurar.

Etimología. El nombre de la especie se propone en alusión a la Reserva de la Biosfera "El Cielo" (RBC) del Estado de Tamaulipas, México, lugar donde fue colectada por primera vez.

La planta aquí descrita se ubica en la serie Proliferae D.R. Hunt, con la cual comparte las siguientes características: la presencia de espinas radiales pequeñas, flexibles, aciculares y nunca uncinadas, las espinas radiales y centrales semejantes (forma, consistencia y color), casi no diferenciables, salvo por el tamaño de las centrales.

M. cielensis difiere de M. picta, M. prolifera, M. vetula y M. zublerae por mostrar espinas radiales de color blanco vítreo y centrales de color blanco, mientras que el resto de las especies tienen otro matiz. Además, varía de este conjunto de plantas por presentar un tallo globoso a oviforme de menor tamaño y una carencia de tricomas en las axilas de los tubérculos. Los frutos son de color rojo a rosado, de menor tamaño (3.5-4 mm de ancho), a diferencia de las otras que los presentan con otras tonalidades de rojo a rojo escarlata y son de mayor talla (9-25 $\mathrm{mm}$ y 4-10 $\mathrm{mm}$ ). Adicionalmente, $M$. cielensis produce semillas más grandes $(2 \mathrm{~mm}$ de largo por 1.7 $\mathrm{mm}$ de ancho), de forma elíptica, así como una testa foveolada muy hundida y ligeramente reticulada, además de un hílum de mayor longitud $(52 \mu \mathrm{m})$, en comparación con las otras especies (ver Cuadro 1).

Mammillaria cielensis muestra mayor semejanza morfológica con M. zublerae, pero difiere de ésta por presentar un tallo globoso a oviforme con ápice redondeado y tubérculos sin tricomas en las axilas. Las espinas radiales y centrales son más numerosas, de color blanco vítreo y de forma acicular. Las semillas son elípticas, con un hílum grande de mayor tamaño $(52 \mu \mathrm{m})$. Por su parte, $M$. zublerae presenta menos espinas radiales y centrales y éstas son ligeramente uncinadas y amarillentas. Las semillas son reniformes con un hílum pequeño $(25 \mu \mathrm{m})$, ventral subbasal (Fig. 2). La nueva especie propuesta ocupa hábitats húmedos con vegetación de bosque de pino-encino (zona núcleo norte), a diferencia de M. zublerae, la cual crece en lugares más secos, en bosque de pino con asociaciones de matorral rosetófilo. 

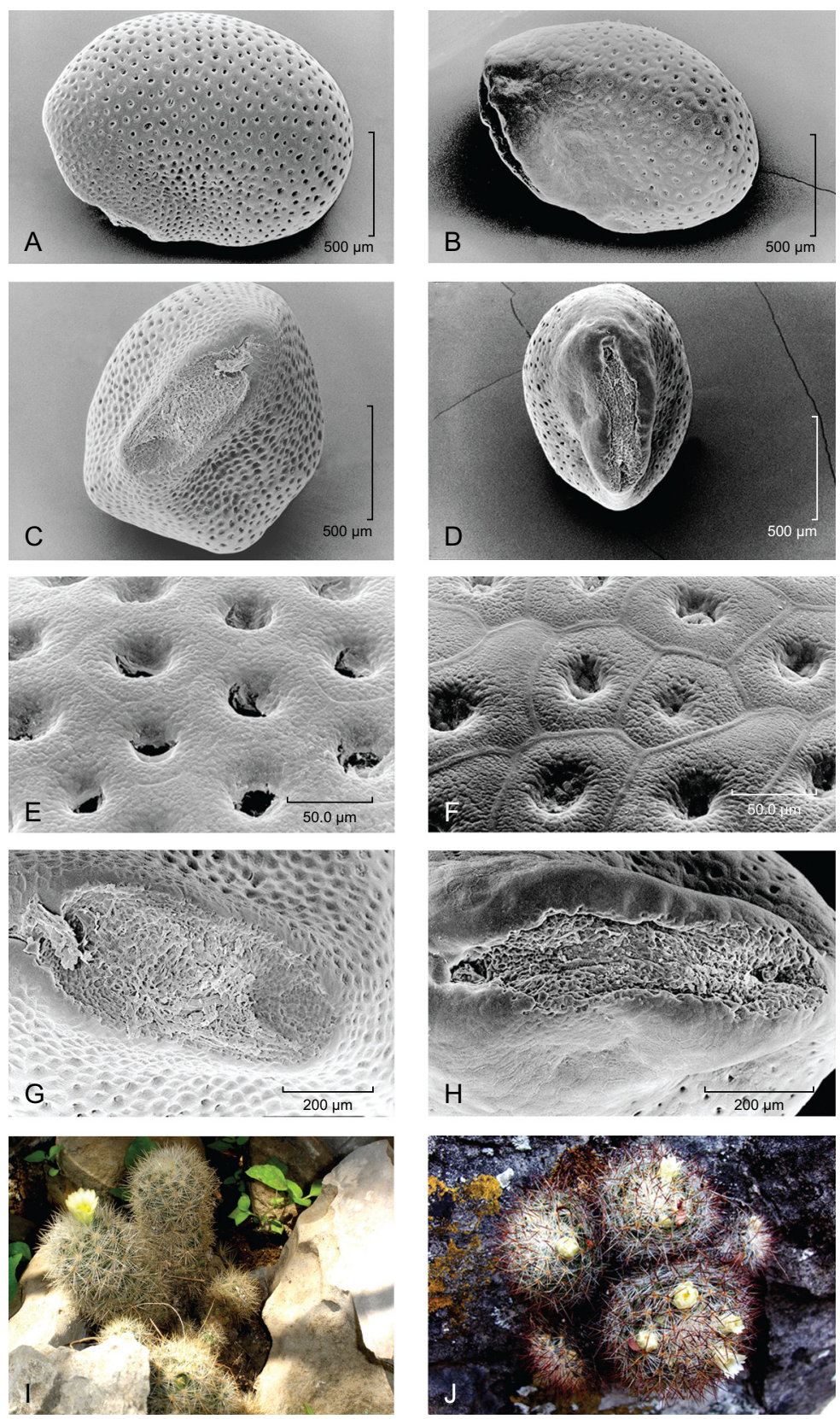

Fig. 2. Morfología de las semillas en el microscopio electrónico de barrido y fotografías de de Mammillaria. cielensis y M. zublerae en sus respectivos hábitats. A, C, E, G, I M. cielensis; $\mathrm{B}, \mathrm{D}, \mathrm{F}, \mathrm{H}, \mathrm{J}$ M, zublerae. 
Martínez-Ávalos et al.: Nueva especie de Mammillaria para Tamaulipas, México

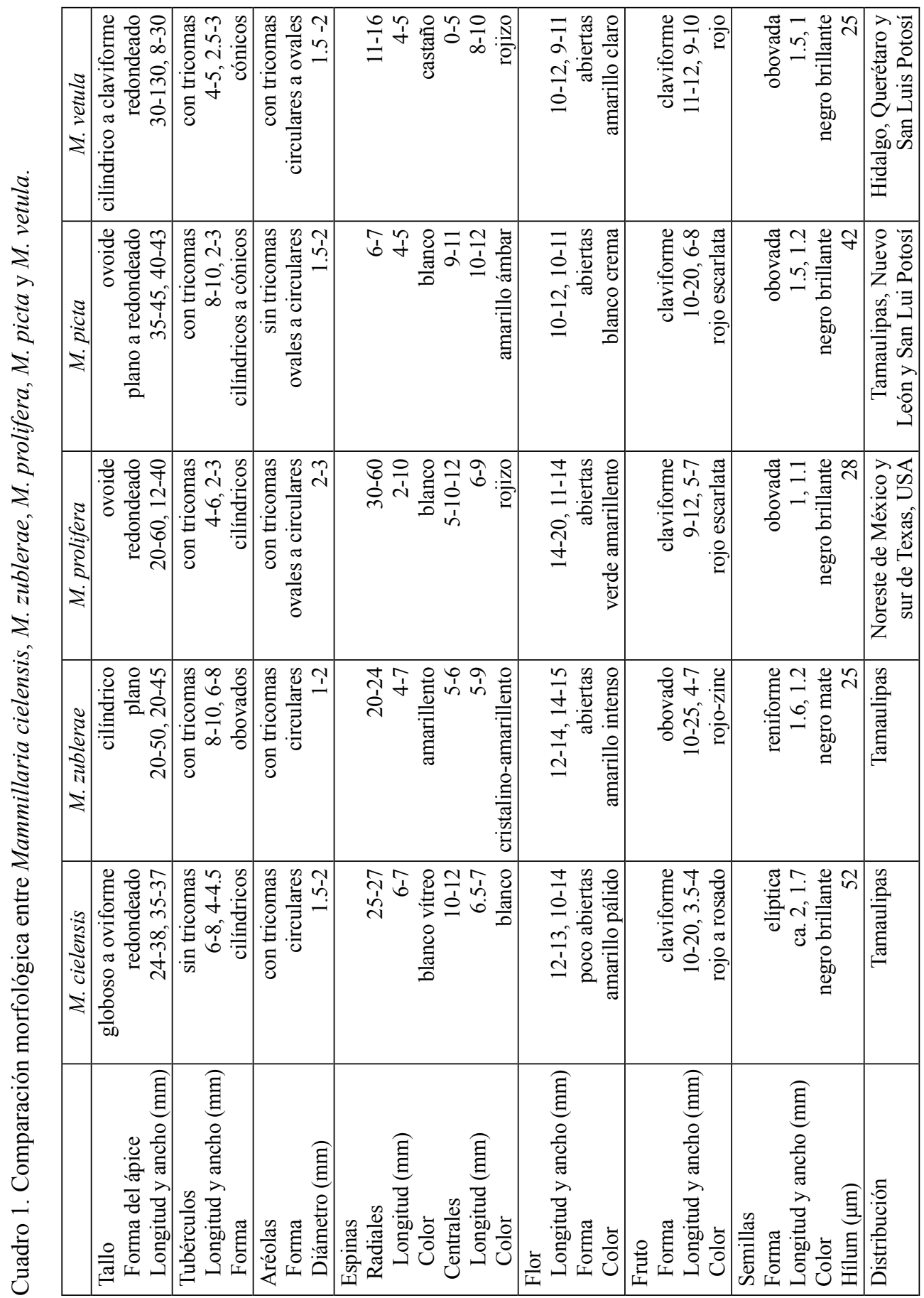


Se presenta a continuación una clave para diferenciar a M. cielensis y especies relacionadas.

A. Axilas de los tubérculos setosas.

B. Espinas (centrales más radiales) menos de 22 M. picta Meinsh.

BB. Espinas (centrales más radiales) más de 30.

C. Tubérculos mayores de $8 \mathrm{~mm}$ de longitud y mayores de $6 \mathrm{~mm}$ de ancho M. zublerae Repp.

CC. Tubérculos menores de $8 \mathrm{~mm}$ de longitud y menores de $6 \mathrm{~mm}$ de ancho.

D. Espinas pubescentes; plantas profusamente cespitosas; tallos de 1.2-4 cm de diámetro .......................................... M. prolifera (Mill.) Haw.

DD. Espinas no pubescentes; planas simples o cespitosas, tallos de $3-7 \mathrm{~cm}$ de diámetro.

E. Tallo de 4-7 cm de diámetro; espina central 1, acompañada de 4-7 subcentrales hacia el ápice de la aréola

M. pilispina J. A. Purpus

EE. Tallo de 3-4 cm de diámetro; espinas centrales 1-4, o ausentes M. albicoma Boed.

AA. Axilas de los tubérculos no setosas.

F. Espinas radiales 25 a 30; espinas centrales siempre presentes; plantas algo cespitosas.

G. Espinas radiales 25 a 27, de 6 a $7 \mathrm{~mm}$ de longitud M. cielensis

GG. Espinas radiales 27 a 30 , menores de $5 \mathrm{~mm}$ de longitud

M. vetula Mart.

FF. Espinas radiales 12 a 16, espinas centrales frecuentemente ausentes; plantas profusamente cespitosas M. gracilis Pfeiff.

\section{AGRADECIMIENTOS}

Se agradece al Biól. Omar González Zorzano por elaborar la diagnosis en latín, a los revisores anónimos de esta revista por sus observaciones, al Dr. Helmut Rogozinski por las facilidades otorgadas para la toma fotográfica en microscopia electrónica de semillas, y al apoyo obtenido del proyecto CONAFOR-CONACYT No. 69919. 


\section{LITERATURA CITADA}

Anónimo. 2001. Diagnóstico ecológico del estado de Tamaulipas. Gobierno del Estado de Tamaulipas. Cd. Victoria, Tamaulipas, México. 267 pp.

Anónimo. 2002. Síntesis geográfica del estado de Tamaulipas. Serie III. Secretaría de Programación y Presupuesto, Instituto Nacional de Estadística, Geografía e Informática. Aguascalientes, Aguascalientes, México.

Bravo, E. H. y H. Sánchez-Mejorada. 1991. Las cactáceas de México. Tomo III. Universidad Nacional Autónoma de México. México, D.F. 643 pp.

Butterworth, C. y R. S. Wallace. 2004. Phylogenetic studies of Mammillaria (Cactaceae) - insights from chloroplast sequence variation and hypothesis testing using the parametric bootstrap. Amer. J. Bot. 91(7): 1086-1098.

Guzmán, U., S. Arias y P. Dávila. 2003. Catálogo de cactáceas mexicanas. Universidad Nacional Autónoma de México-Comisión Nacional para el Conocimiento y Uso de la Biodiversidad. México, D.F. 315 pp.

Hunt, D. 1997. Mammillaria. Postcripts 6: 1-20.

Hunt, D., N. P. Taylor y G. Charles. 2006. The new cactus lexicon. DB Hunt Books. Milborne Port, UK. 373 pp.

Lüthy, J. M. 1995. Taxonomische Untersuchung der Gattung Mammillaria Haw. Tesis de doctorado. Universität Bern. Bern, Suiza. 230 pp.

Lüthy, J. M. 2001. A revised classification of the "primitive" mammillarias. J. Mammillaria Soc. 41: 6-7.

Martínez-Ávalos, J. G., H. Suzán, J. Jiménez, A. Mora, H. Hernández y A. P. Vovides. 1996. Le Cactaceae della Riserva della Biosfera "El Cielo". Piante Grasse 16(6): 82-91.

Pilbeam, J. 1999. Mammillaria.The cactus file. Handbook No. 6. Cirio Publishing Services. Ltd. Southampton. $376 \mathrm{pp}$.

Puig, H. y R. Bracho. 1987. El bosque mesófilo de montaña del estado de Tamaulipas. Instituto de Ecología, A.C. Xalapa, Ver. 179 pp.

Reppenhagen, W. 1991-1992. Die Gattung Mammillaria. Monographie. Druckerei Steinhart. Titisee-Neustadt, Alemania. 2 vols. 